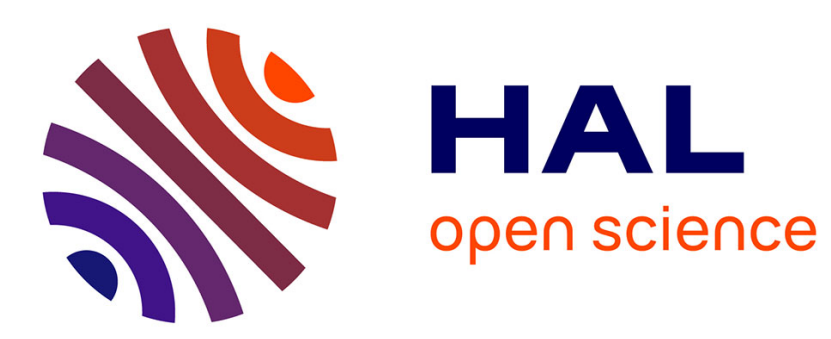

\title{
Faire entendre la parole des citoyens par le recours au film. Analyse d'un panel de citoyens dans la gestion de l'eau. \\ Laura Seguin
}

\section{- To cite this version:}

Laura Seguin. Faire entendre la parole des citoyens par le recours au film. Analyse d'un panel de citoyens dans la gestion de l'eau.. Participations - Revue de sciences sociales sur la démocratie et la citoyenneté, 2013, Photographie et film: antidotes à la domination politique ?, 3 (7), pp.127-149. 10.3917/parti.007.0127 . halshs-01324885

\section{HAL Id: halshs-01324885 \\ https://shs.hal.science/halshs-01324885}

Submitted on 8 Aug 2016

HAL is a multi-disciplinary open access archive for the deposit and dissemination of scientific research documents, whether they are published or not. The documents may come from teaching and research institutions in France or abroad, or from public or private research centers.
L'archive ouverte pluridisciplinaire HAL, est destinée au dépôt et à la diffusion de documents scientifiques de niveau recherche, publiés ou non, émanant des établissements d'enseignement et de recherche français ou étrangers, des laboratoires publics ou privés. 


\title{
Faire entendre la parole des citoyens par le recours au film. Analyse d'un panel de citoyens dans la gestion de l'eau
}

\author{
Laura Seguin \\ Version auteure
}

Comment expliquer que, malgré le bon déroulement d'une procédure participative et un travail de qualité produit par les citoyens sollicités pour donner leur avis, la prise en compte de cet avis dans le système décisionnel ne soit pas assurée ${ }^{1}$ Que se passe-t-il après la fin d'une conférence de citoyens, dans ces moments où se construit la décision ? Il existe là un espace charnière encore peu exploré, dans lequel le travail produit par les citoyens, mais aussi l'expérience participative dans sa globalité, doivent être traduits pour être restitués aux commanditaires, voire publicisés plus largement. Lors de ces moments de restitution, les « instruments matériels et humains » de «traduction » (Callon, 1984; Lascoumes, 2010) jouent un rôle essentiel. Ce sont eux qui rendent visible ou audible ce qui s'est passé lors de ce huis-clos que constitue souvent le dispositif participatif, ainsi que son résultat final, qui prend la plupart du temps la forme d'un avis. Or les instruments de mise en visibilité des discours politiques sont des enjeux forts de pouvoir, distribués et maîtrisés de façon asymétrique entre "citoyens ordinaires » et professionnels de la politique, participant ainsi aux effets de domination politique. Cet article propose de répondre à la question de l'inclusion d'un avis citoyen dans le système décisionnel par l'analyse des instruments de traduction employés. Le choix des outils matériels de traduction et la façon dont ils sont utilisés ne sont pas neutres et doivent être pris en compte dans l'analyse de ce qui se joue dans la boîte noire de la « restitution » d'une démarche participative.

Nous appréhenderons un instrument de traduction original qu'est l'outil audiovisuel, utilisé lors de la restitution d'une procédure participative dans le domaine de la gestion de l'eau. Il s'agit d'un "panel d'habitants $»^{2}$ mis en place en 2011 par l'Établissement public territorial de bassin du fleuve Charente (EPTB Charente), en partenariat avec l'Institut de formation et de recherche en éducation à l'environnement (Ifrée) en Poitou-Charentes. Ce panel d'habitants devait répondre à la question suivante : «Comment concilier les différents usages de l'eau avec le bon état des eaux sur le bassin de la Charente ? » Une fois la procédure terminée, l'EPTB Charente et l'Ifrée ont préparé, avec les membres du panel, l'étape importante de restitution de l'avis produit auprès de la Commission locale de l'eau (CLE) du bassin versant de la Charente. Précisons ici la configuration particulière de cette procédure : l'EPTB Charente, commanditaire de la démarche, n'est pas la structure décisionnelle à même de prendre en compte l'avis du panel. C'est en effet à la CLE de décider si, dans ses futures délibérations visant à élaborer le Schéma d'aménagement et de gestion des eaux (SAGE) ${ }^{3}$,

\footnotetext{
${ }^{1}$ Je tiens à remercier le comité de rédaction de ce numéro, Héloïse Nez et Cécile Cuny, pour leur aide précieuse, ainsi que les relecteurs de la revue pour leurs commentaires et leurs conseils dans la rédaction de cet article.

${ }^{2}$ Le panel d'habitants est une procédure « mini-public » impliquant des citoyens « profanes » sur l'objet débattu recrutés par tirage au sort et offrant les conditions nécessaires à la délibération et à la rédaction collective d'un avis (Bourg et Boy, 2005 ; Goodin et Dryzek, 2006 ; Sintomer, 2007).

${ }^{3} \mathrm{La}$ CLE est l'instance décisionnelle sur un bassin versant, chargée d'élaborer un document de planification de la gestion de l'eau et des milieux aquatiques : le SAGE.
} 
elle tiendra compte ou non de cet avis. Certes, l'EPTB Charente est la structure porteuse de la CLE, mais cette dernière possède son autonomie, avec son propre bureau présidé par un élu local. C'est donc dans ce contexte particulier que prend place l'étape de restitution.

J'ai réalisé un travail d'enquête tout au long de cette démarche, des réunions de préparation en amont, jusqu'au suivi de l'avis citoyen dans la construction de cette politique locale de l'eau. En 2011, j'ai occupé le statut de stagiaire au sein de l'Ifrée pour accompagner la préparation et la mise en place du panel, et porter un regard réflexif et évaluatif sur cette expérimentation. L'évaluation a été co-construite avec les participants, par le biais d'entretiens venant enrichir mes observations. Ce travail d'enquête ethnographique conjuguait donc observation participante et entretiens semi-directifs, à la fois auprès des acteurs engagés dans la mise en place de la démarche et auprès des participants au panel. À la fin de la procédure, je me suis plus particulièrement penchée sur les instruments de restitution et de valorisation de cette démarche. Il s'agit de deux films aux contenus et aux finalités différents.

Le premier film abordé dans cet article a été produit par les commanditaires de la procédure dans le but de restituer l'avis citoyen auprès de la CLE. Il s'agit d'une lecture de l'avis par le panel d'habitants qui l'a élaboré, précédé d'un mot des présidents des deux structures commanditaires et d'une présentation de la démarche. Ce film sera analysé comme outil de traduction et de médiation de la parole citoyenne, mais aussi comme instrument jouant un rôle dans le maintien de la distinction des arènes et des rôles politiques. Le second est un film de recherche que j'ai réalisé à partir d'entretiens avec des participants, tourné quelques mois après la fin du panel ${ }^{4}$. Ce film restitue l'expérience telle qu'elle a été vécue, ainsi qu'un regard réflexif sur celle-ci. Nous analyserons les effets que la visualisation du film a pu avoir, d'une part chez les participants au panel, d'autre part chez les commanditaires de la démarche. L'objectif de cet article est ainsi d'explorer les enjeux de l'utilisation de l'outil audiovisuel dans la restitution d'une expérience participative, lorsque celui-ci est mis au service de la parole citoyenne.

\section{Entre mise en valeur de la parole citoyenne et maintien des rôles politiques}

S'interrogeant sur le support à utiliser pour la restitution de l'avis lors de la réunion de la CLE, l'EPTB et l'Ifrée validèrent conjointement l'idée d'un film présentant à la fois la procédure et l'avis produit par les citoyens. Ce choix s'explique principalement par la volonté de donner plus de visibilité à la démarche et de rendre la présentation plus vivante et dynamique dans un ordre du jour chargé. Huit mois après la fin de la procédure, les membres du panel ont donc été à nouveau sollicités durant une journée de tournage.

\section{Faire passer « du bruit à la parole »}

Les professionnels de la participation ont de plus en plus recours au film pour faire entendre la «parole» citoyenne ou pour mettre en valeur les procédures participatives. Il intervient pour assurer la «monstration » (Bonaccorsi, Nonjon, 2012) du résultat final, mais également de l'« ingénierie participative » (Gourgues, 2012, p. 7) $)^{5}$. On peut ainsi s'interroger sur son rôle dans le «tropisme normatif et procédural » (Mazeaud, 2012, p. 54) caractéristique des

\footnotetext{
${ }^{4}$ Ce film, intitulé Paroles de citoyens. Récit d'une expérience participative dans le domaine de l'eau, est consultable en annexe électronique de cet article sur le site de la revue Participations

5 Pour un autre exemple d'outil visuel de «monstration », voir la bande-dessinée « Métropole en ébullition » restituant la démarche prospective et participative « Amiens $2030 »$.
} 
recherches sur la participation comme de l'action publique qui la met en œuvre. Il est en effet frappant de constater l'attention accordée au perfectionnement des procédures, dont l'ingénierie se veut toujours plus innovante mais également de plus en plus normée. En tant qu'instrument efficace de circulation, le film n'a-t-il pas une influence dans la construction d'une « offre institutionnelle de dispositifs de participation publique » (Gourgues, 2012, p. 6) de plus en plus standardisée ${ }^{6}$ ?

Lors de la préparation du panel, l'équipe de l'Ifrée avait visionné des vidéos sur la mise en place de conférences de citoyens. À l'issue de la démarche, le choix du film permet aux commanditaires de montrer à leur tour la procédure réalisée et l'avis produit. Ce film a d'ailleurs rapidement été pensé comme un support de communication, avec notamment une introduction par les présidents des structures commanditaires. Mais devant les soupçons de récupération politique exprimés par les panélistes au début de la journée de tournage, les commanditaires s'engagèrent finalement à l'utiliser uniquement lors de la réunion de la CLE. Le film garda malgré tout l'aspect d'un support de communication : les parties introductives présentant la démarche et les deux partenaires furent conservées. Le début de la journée de tournage fût donc marqué par de fortes réticences : certains refusèrent d'être filmés malgré l'engagement des commanditaires, d'autres acceptèrent en tant que figurant, mais non en tant que lecteur de l'avis ${ }^{7}$. Figurer dans un film institutionnel à usage potentiellement public peut en effet être source d'inquiétudes. Plus fortement que l'avis en version papier, le film rend visible des prises de position qui n'ont au départ pas été exprimées dans la perspective d'une publicisation plus large. Fabrice explique ainsi son refus d'être filmé : «Je ne veux pas que ma parole ait un visage, je suis ni agriculteur, ni... Je suis juste un citoyen qui essaie d'apporter une réflexion, la meilleure possible, juste en tant qu'être humain. ${ }^{8}$ Or la mise en film contribue à personnaliser un avis qui n'apparaît alors plus comme «la parole des citoyens », mais comme « la parole de ces citoyens-là » à laquelle sont associés des visages et des voix.

Après ce temps de négociations, le tournage put commencer. Pour illustrer le travail du panel, la réalisatrice 9 a mis les participants en scène en train de lire, écrire, surligner des documents et discuter ensemble comme ils l'ont fait pendant la procédure. Le rôle de l'animateur de l'Ifrée se trouve également illustré, lorsqu'on le voit donner des explications tout en pointant du doigt l'écran sur lequel est projeté un document de travail. Ces images défilent alors qu'il expose en voix off les principes et le déroulement de la procédure. Après cette brève présentation, le reste du film est uniquement consacré à la lecture de l'avis final par les citoyens. Sur leur lecture ont été ajoutées des images de l'environnement naturel (fleuve, rivières, marais, etc.), ainsi que des différents usagers de l'eau du bassin. Ces images suggèrent l'emprise de l'homme sur son environnement, son rapport à l'eau, et illustrent l'esprit de l'avis.

\footnotetext{
${ }^{6}$ Sur ce point, voir les enquêtes audiovisuelles de Campana Eleb Sablic, agence ayant joué un rôle dans l'« homogénéisation des pratiques participatives » de municipalités communistes en Île-de-France (Nez, Talpin, 2010, p. 106).

${ }^{7}$ C'est le cas de Georges, l'agriculteur du groupe, le plus critique vis-à-vis de l'avis final.

${ }^{8}$ Compte-rendu d'observation de la journée de tournage, 18-02-2012.

9 Animatrice des Jardins d'Isis, association locale d'éducation à l'environnement. L'EPTB a fait appel à elle sur les conseils de l'Ifrée et a entièrement financé la réalisation du film.
} 


\section{Captures d'écran du film : journée de tournage et berges artificialisées en bord de Charente}
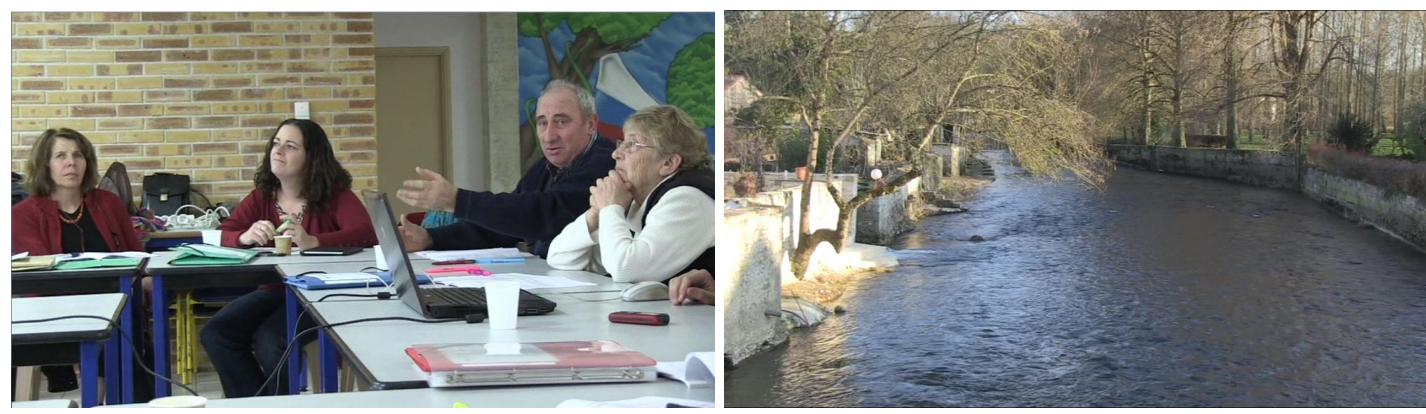

(C) EPTB Charente

Au-delà des images, c'est la lecture de l'avis, la parole, qui est le fil conducteur du film. Mais de quelle parole s'agit-il ? Les démarches participatives sont censées rendre audible la voix de ceux à qui est habituellement déniée la légitimité de prendre part aux décisions. Ici, c'est la parole du "public », traditionnellement distingué des "parties intéressées » dans le langage des politiques de l'eau (Notte, Salles, 2011, p. 44), qui était recherchée. Le tirage au sort, utilisé pour recruter le panel, avait été ajusté pour constituer un groupe qui soit «le plus éloigné possible de la logique d'acteurs engagés et organisés sur la question de l'eau ${ }^{10}$. La seule parole formalisée de ce «public» jusqu'alors entendue par les acteurs de l'eau interrogés était celle issue des sondages d'opinion, dont ils reconnaissaient les travers et regrettaient la pauvreté des réponses : "Ça reste un peu primaire, voire primitif »" Il en ressort une impression de «bruit » diffus dont on ne sait pas vraiment quoi faire. Car pour être légitimement entendue, cette parole des citoyens aujourd'hui tant recherchée doit prendre des formes « policées », c'est-à-dire entrer dans l'espace de la "police » qui définit des «modes du dire » acceptables et d'autres non acceptables, selon les termes de Jacques Rancière : « La police $[. .$.$] est un ordre du visible et du dicible qui fait que telle activité est visible et que telle$ autre ne l'est pas, que telle parole est entendue comme du discours et telle autre comme du bruit » (1998, p. 60). La procédure visait ainsi à faire passer un groupe d'habitants profanes en matière de gestion de l'eau « du bruit à la parole » (Anselme, 2000). Le film montre le résultat de ce processus de production d'une parole, celle des citoyens ${ }^{12}$, à partir d'une multiplicité de voix contradictoires, imprécises, mouvantes. L'avis est construit, la rédaction est travaillée, les propositions sont nourries de données précises, d'un point de vue critique sur celles-ci et de considérations politiques argumentées :

«De nombreuses données [sur l'état de l'eau] sont partielles voire inexistantes. Ainsi, l'état chimique de près de la moitié des masses d'eau de surface du bassin de la Charente n'est pas suivi. Comment alors identifier finement les zones où apparaissent

\footnotetext{
${ }^{10}$ Extrait d'un document de travail de l'Ifrée. Lors du recrutement, une secrétaire dans une entreprise d'irrigation et une gestionnaire de l'EPTB Charente initialement tirées au sort ont été écartées. La volonté de ne recruter que des «non initiés » ou «non engagés » a des limites : trois personnes sur les douze étaient partiellement initiées voire engagées sur les problèmes de l'eau et de l'agriculture intensive sur le territoire. De plus, il est difficile d'évaluer a priori le degré de politisation des individus recrutés : deux personnes avaient une expérience d'engagement associatif ou syndical (cependant non liée à l'eau).

${ }^{11}$ Entretien avec un représentant de l'Agence de l'eau Adour-Garonne, membre du comité de pilotage de la démarche, 07-02-2011.

${ }^{12}$ Cf. titre de l'avis final : «Concilier les différents usages avec le bon état des eaux sur le bassin de la Charente : ce qu'en disent les citoyens ».
} 
les pollutions chimiques et rechercher les causes de ces pollutions? Nous nous interrogeons également sur la réalité des données, la neutralité des chiffres. On sait que leur interprétation est parfois difficile et dépend fortement des intérêts de celui qui la fait [...] Or la connaissance du fonctionnement des systèmes incluant l'eau (de surface et de profondeur), les sols et leurs types d'occupation, ainsi que les usages de l'eau, est déterminante pour définir les mesures à prendre en matière de gestion de l'eau. ${ }^{13}$

Le film donne finalement à voir un des principes à l'origine de toute démarche participative basée sur la délibération, selon lequel le contexte, le « facteur situationnel », est déterminant dans la construction de la compétence politique :

«Si les individus sont parfois incapables de fournir autre chose que des opinions noninformées, incohérentes et "top of the head", sur des sujets auxquels ils n'ont jamais véritablement réfléchi jusqu'alors, il suffit de les placer - ou de les observer - dans des circonstances favorables pour qu'ils formulent des raisonnements politiques relativement sophistiqués » (Talpin, 2010, p. 96-97).

Reformulé dans les termes de l'Ifrée : quand on met les citoyens dans une «relation de confiance » et dans de «bonnes conditions d'information et de réflexion », ils sont capables de produire de l' ' intelligence collective $»{ }^{14}$.

\section{Dans les coulisses du tournage : une parole «trop bien élevée »?}

Ces réflexions amènent nécessairement à interroger le processus par lequel ces multiples opinions informées, floues, approximatives, sont devenues en l'espace de trois week-ends une parole quasi experte, précise, argumentée. La procédure a évidemment favorisé la montée en expertise grâce aux apports d'informations et aux auditions d'acteurs et d'usagers de l'eau. Pour favoriser l'appropriation et la mise en commun des données, des «séquences d'intégration » ont été aménagées tout au long du processus. Elles consistaient à collecter et synthétiser les informations à différentes étapes et à identifier des éléments de réponse à la question posée. La délibération et la rédaction de l'avis ont également fait l'objet d'un accompagnement. L'équipe d'animation joue donc un rôle majeur dans ce type de procédure, accompagnant le passage de l'approximatif au précis, de la pluralité des voix à l'univocité. En effet, il est important de noter qu'il ne s'agit pas de «l'avis du panel», mais de l'avis du panel « médié » par le travail des animateurs :

«Sans mettre aucunement en cause leur déontologie ni transformer les panélistes en proies faciles, force est cependant de constater que, à côté du "bon sens non spécialisé" des panélistes et de la qualité des procédures, les professionnels de la participation doivent clairement être comptés au nombre des co-producteurs de l'avis final » (Barbier, Bedu, Buclet, 2009, p. 198).

C'est au moment de la rédaction que cette co-production est la plus problématique : à partir de quel degré d'intervention des animateurs peut-on dire que l'avis n'est plus véritablement celui des citoyens? Les animateurs sont à compter parmi les rédacteurs de l'avis, puisqu'ils ont effectué un important travail de formulation, de précision de ce qui avait été rédigé lors du dernier week-end. À partir de ce premier document, ils ont proposé aux panélistes une deuxième rédaction "améliorée ", qui pouvait être amendée à distance (par courrier,

\footnotetext{
${ }^{13}$ Transcription de la vidéo : lecture de l'avis.

${ }^{14}$ Entretien avec le président de l'Ifrée, 16-05-2012.
} 
téléphone ou courriel). Puis une séance collective supplémentaire a été programmée, afin de recueillir les derniers commentaires et de valider définitivement cette troisième et ultime version. Leur travail, qui a surtout consisté en une valorisation de l'écrit, pour le légitimer aux yeux de ses lecteurs ${ }^{15}$, a pu créer un certain décalage entre le premier écrit (relevant plutôt du langage parlé, informé) :

- «Adapter les activités humaines au type de sol : ne pas mettre certaines activités (agricoles et industrielles) dans des zones où les sols ne sont pas adaptés.

- Favoriser l'élevage dans les marais. »

Et ce qui figure dans l'avis final (registre du langage écrit, de l'argumentation) :

«Adapter les activités humaines au type de sol présent afin de protéger certains milieux naturels des conséquences d'activités menaçant directement la qualité de la ressource en eau. Il semble notamment indispensable :

- de stopper le remblai de zones humides à des fins d'installation d'activités industrielles ou commerciales ;

- de favoriser des activités appropriées au milieu naturel et à l'écosystème propres au marais, comme par exemple l'élevage. »

Cette première «traduction » a introduit un décalage particulièrement visible lors du tournage du film, qui constitue un deuxième processus de traduction. Au-delà de la présence de la caméra qui a pu être dérangeante, il était frappant de constater les difficultés qu'ont rencontrées les participants dans un exercice en apparence simple : la lecture de leur avis. Car cette double traduction ne constitue pas au final un retour en arrière (parlé - écrit - parlé) : le «parlé » final se trouve être radicalement différent du premier, épousant difficilement les formes de l'écrit. La journée fut longue, l'exercice de lecture fastidieux, ponctué d'erreurs, de bafouillages, de difficultés à comprendre certaines phrases. Des participants ont plaisanté en constatant la qualité de la rédaction : "Mais c'est vraiment nous qui avons écrit ça ? On était dopés, ce n'est pas possible ! »"${ }^{16}$

Si cette lecture apparaît aussi difficile, c'est également parce qu'entre le moment de la rédaction de l'avis et celui du tournage du film, huit mois se sont écoulés, pendant lesquels les participants ont probablement oublié des passages de l'avis. Leur réflexion a également pu évoluer, certains ne se reconnaissant plus tout à fait dans l'avis final. Celui-ci contribue effectivement à réifier un processus de débat complexe et ne reflète finalement qu'un moment de la délibération. Entre la dynamique réelle de l'argumentation et l'écrit final, l'écart est considérable, comme le montre Clémence Bédu (2010, p. 34) en repérant les différentes «épreuves de traduction» des énoncés au sein d'une «communauté débattante ». De nombreux filtres sont à l'œuvre entre la première expression d'un énoncé et sa forme finale dans l'avis : il peut être disqualifié au profit d'un autre lors d'une délibération en petit groupe, puis connaître un nouvel élan lors de la mise en commun en séance plénière, se trouver modifié, amendé, passer du statut d'argument à celui de constat, et finir par rencontrer un accord collectif pour figurer dans l'avis. Dans le film, ce long itinéraire délibératif se déployant dans une discontinuité temporelle est restitué en quelques secondes. L'exercice final a imposé aux citoyens de se mettre d'accord en peu de temps sur des propositions. Les questionnements, les nuances et les détails faisaient partie d'un registre de langage à éviter.

\footnotetext{
${ }^{15}$ C'est-à-dire principalement aux yeux de ses commanditaires (élus et techniciens de l'eau) et des membres de la CLE qui auront à prendre en compte l'avis (élus, usagers, services de l'État, collectivités territoriales, etc.).

${ }^{16}$ Compte-rendu d'observation de la journée de tournage, 18-02-2012.
} 
Les principales interventions des animateurs consistaient alors à couper court aux débats et à inciter à rédiger les propositions. De plus, le format imposé par le film a obligé les panélistes à effectuer des «coupures » dans l'avis, celui-ci ne pouvant être lu intégralement en 25 minutes ${ }^{17}$. Le tournage a débuté par une identification des passages «les moins utiles, les moins intéressants » [animateur Ifrée] ${ }^{18}$, pouvant être coupés sans perdre pour autant le sens de l'avis. Ce sont à nouveau les détails, les parenthèses informatives qui ont alors été éliminés : la plupart des coupes se situent dans les paragraphes de « constats » introduisant les « propositions ». La mise en film a donc accentué les propositions et a rendu moins visible le travail d'appropriation et de synthèse des informations qui les a nourries.

Étant donnés les objectifs des commanditaires, hésitations et bafouillages n'ont pas été retenus lors du montage. Le film donne donc l'impression que l'avis a été approprié, alors que le tournage montre à l'inverse combien cette appropriation a été incertaine, le registre argumentatif durement construit et surtout co-construit avec les animateurs. L'avis est finalement un objet mouvant et peu consensuel : certains participants souhaitaient revenir sur ce qui avait été validé en séance finale, d'autres effacer certaines propositions, d'autres encore y faire des ajouts. L'observation des coulisses du tournage s'est donc avérée particulièrement instructive sur les limites de telles procédures. Leur caractère parfois trop procédural et réglé peut faire l'objet de critiques et interroge «l'apport de ces débats publics "d'élevage" (suscités, organisés, institués), par rapport aux débats publics "sauvages" (autonomes, spontanés, "ingérables") » (Mermet, 2007, p. 370). Dans notre cas d'étude, la parole n'est-elle pas finalement trop « bien élevée » pour être véritablement appropriée ?

\section{L'usage du film : un outil au service du maintien des rôles politiques}

Pour comprendre si ce film a réellement été un outil au service des citoyens, il s'agit désormais d'analyser les modalités concrètes de son usage lors de la restitution devant la CLE. Cette étape, rompant avec le huis clos de la procédure, confronte les participants aux « experts » du sujet sur lequel ils ont débattu pendant quelques jours et les rappelle inévitablement à leur statut de «profanes » ${ }^{19}$. En ce sens, le choix du support audiovisuel permet de ne pas mettre en difficulté les citoyens, en leur évitant d'être directement interpellés par les membres de la CLE sur le contenu de leur avis. Les panélistes avaient exprimé cette inquiétude, doutant de leur capacité à entrer dans un débat avec les membres de la CLE :

« Ça va être compliqué je pense, parce que moi je ne maîtrise pas le sujet. Je n'ai pas assez de recul, pas assez de données, je ne connais pas le métier donc on pourra me coincer dans tous les coins, on pourra me dire "t'y connais rien" [...]. Je n'ai pas envie de passer pour un imbécile. Et puis ce n'est pas mon métier de parler, c'est les politiques qui font ça, ils apprennent à parler, ils préparent leur truc, ils ont les arguments... Donc moi ça me fait un peu peur oui. $»^{20}$

La prise de parole sur le contenu de l'avis était donc anticipée comme une prise de risque. Les participants redoutent l'expérience de la dissymétrie des savoirs et des rapports de pouvoir.

\footnotetext{
${ }^{17}$ Cette durée a été calibrée selon le temps réservé à la présentation du panel lors de la réunion de la CLE : une heure et quart au total. Le film ne devait pas dépasser 25 minutes, afin d'assurer un temps plus long d'échanges entre panélistes et membres de la CLE à l'issue de la projection.

${ }^{18}$ Compte-rendu d'observation de la journée de tournage, 18-2-2012.

${ }^{19}$ Les participants au jury de citoyens sur la gestion des déchets à Saint-Brieuc ont été mis en difficulté lors de la restitution, confrontés au déni de légitimité de la part des acteurs associatifs (Barbier, Bedu, Buclet, 2009).

${ }^{20}$ Entretien avec un membre du panel, 14-11-2011.
} 
D'un côté des savoirs experts, techniques, scientifiques, portés par des acteurs de la gestion de l'eau rompus à l'exercice de l'argumentation et maîtrisant également les enjeux sociaux, économiques et politiques du territoire. De l'autre, des « savoirs d'usage », " ordinaires » ou encore « citoyens » (Sintomer, 2008 ; Topçu, Cuny, Serrano Velarde, 2008 ; Nez, 2011), acquis dans les différentes sphères de la vie sociale des individus. Ces savoirs ont certes été enrichis durant la procédure, mais celle-ci n'a évidemment pas transformé les citoyens en experts de la question. Indirectement, le recours au film permet d'éviter la confrontation entre ces différents types de registres et de savoirs : en lui déléguant la parole, on dépasse l'obstacle de la compétence à argumenter.

Mais le recours au film répondait aussi à une préoccupation de l'EPTB : anticipant l'absence de certains membres du panel à la réunion de la CLE, les initiateurs de la démarche se demandent de quelle manière quelques participants seulement pourraient restituer la parole du groupe. Après huit mois écoulés depuis la fin du panel, chacun a eu l'occasion de poursuivre la réflexion sur le sujet, de nuancer certaines positions prises dans le cours de la démarche, parfois jusqu'à ne plus se reconnaître dans l'avis final. Le caractère collectif de cette parole se révèle donc fragile et éphémère, et le film a pu apparaître comme un moyen de le restituer tout en le figeant. De cette façon, le commanditaire se protège de l'incertitude et d'éventuels " débordements » de la part de participants qui ne se reconnaîtraient pas dans tout ou partie de l'avis et qui viendraient perturber le déroulement de la réunion. Interrogé sur cette question, le technicien de l'EPTB fait référence à Georges, qui avait scandé sur un ton provocateur : «Ah oui, je vais venir à la CLE, je veux les voir tous ces gens-là ! » ${ }^{21}$ Dès lors, l'ensemble de la réunion est pensée comme un moment d'information visant à éviter le débat, la controverse, voire le conflit. Il était clair que les participants au panel «assistaient» aux débats de la CLE mais n'y «participaient » pas. En témoignent le courrier d'invitation envoyé aux panélistes, ainsi que ces propos d'un technicien de l'EPTB :

«Il faudra qu'on soit clair, et bien préciser les règles : les gens qui sont membres de la CLE participent au débat, les gens qui assistent à la CLE y assistent en tant que spectateurs et ne participent pas [...]. C'est à nous de préciser les règles de fonctionnement, en disant attention, ça reste quand même les travaux de la CLE, ce n'est pas la foire quoi ! ${ }^{22}$

Les panélistes y assistent donc en tant que spectateurs et sont autorisés à prendre la parole seulement lorsque les membres de la CLE leur posent des questions. Leur parole sur le fond du débat n'apparaît dès lors que figée dans le film. Peut-on conclure à une réelle expérience de participation, lorsque la restitution à l'instance décisionnelle ne positionne les citoyens qu'en simples spectateurs devant rester extérieurs au débat? Ici, l'instrument de traduction n'est pas mis au service de la «mise en relation des acteurs» et de la «production de significations communes » (Lascoumes, 2010, p. 636) entre citoyens et élus, profanes et experts. Cette expérience de restitution révèle ainsi la claire séparation des espaces de débat: le premier, celui des panélistes, qui prend le temps de l'apprentissage et de la réflexion, mais qui n'a pas de pouvoir décisionnaire ; le second, l'arène de la CLE, à qui l'on souhaite faire connaître l'expérience et l'avis, sans pour autant perturber son fonctionnement. Comme l'indique Loïc Blondiaux :

« Tout dispositif participatif instaure par ailleurs un cadrage relativement strict des places occupées par les acteurs, qui maintient le "citoyen ordinaire" ou "profane" dans

\footnotetext{
${ }^{21}$ Compte-rendu d'observation de la journée de tournage.

${ }^{22}$ Entretien avec un technicien de l'EPTB Charente.
} 
un rôle et des limites fixées à l'avance, souvent matérialisées dans les lieux mêmes de la discussion » (2008, p. 139).

Toutefois, il ne faut pas exagérer le rôle du cadrage imposé par le dispositif. D'une part, les citoyens ne sont pas tout à fait étrangers à ce jeu d'attribution des rôles. Nous avons vu que le film leur permettait d'éviter l'inconfort légitime que représenterait une confrontation sur le fond de l'avis avec les membres de la CLE. D'autre part, le recours au film leur a indirectement permis de s'exprimer dans un tout autre registre. Ils ont en effet trouvé dans le moment d'échange avec les membres de la CLE un espace pour exprimer une parole à travers laquelle ils ne se positionnent plus en tant que spectateurs, mais en tant que "citoyens vigilants » (Rosanvallon, 2006, p. 64) et juges de la politique qui sera construite par cette commission. En témoignent ces extraits d'échanges :

«Président de la CLE : Vous dressez un bilan favorable de cette expérience ?

Marie : Je crois qu'on pourra juger si effectivement il y a un aspect positif, un résultat à l'issue du SAGE, si vraiment on arrive à retrouver un bon état de l'eau à long terme.

Paul : Je crois qu'on s'est tous investis à fond dans ce qu'on a fait [...] Mais j'espère surtout que vous serez responsables, parce que la santé n'a pas de prix, que vous prendrez des décisions en pensant à vos enfants et à vos petits-enfants, et non pas à vos intérêts financiers, c'est d'abord la santé qui compte. ${ }^{23}$

Le film apparait ici comme un instrument répondant de façon plus ou moins détournée aux préoccupations et aux craintes des citoyens comme des acteurs politiques. En ce sens, il semble jouer un rôle dans la reproduction de la dissymétrie des relations de pouvoir à l'œuvre dans les procédures dites participatives. Mais s'il ferme le débat sur l'enjeu politique que représente l'eau, il contribue à ouvrir un autre espace de politisation des discours. En déléguant la parole argumentative au film, les citoyens ont pu se sentir plus à même de s'exprimer sur un autre registre, celui de l'avertissement ou du jugement, et de s'ériger ainsi en citoyens vigilants, voire défiants ${ }^{24}$ à l'égard du travail des élus. L'attente de résultats et l'injonction à la responsabilité exprimée par les panélistes sont des actes de langage forts dans cette arène où des enjeux environnementaux et sociaux d'importance sont débattus.

\section{Le film de recherche : une confrontation à l'évaluation et à la réflexivité citoyenne}

Puisqu'aucun espace n'avait permis, au sein du processus institué, de confronter les commanditaires à l'évaluation qu'en faisaient les participants, la démarche de recherche devenait potentiellement un lieu d'expression des points de vue, des réactions, des sentiments. Dans cette optique, l'outil audiovisuel m'est apparu comme un bon moyen de traduire cette parole "évaluative » auprès des commanditaires. J'ai donc réalisé un film de recherche s'inscrivant dans la continuité du travail d'évaluation de la démarche initié lors de mon stage au sein de l'Ifrée. Cette posture particulière du chercheur travaillant sur une démarche participative, tout en y étant engagé, pose évidemment la question d'une juste mesure à trouver entre engagement dans l'action et distance critique. Elle offre des avantages

\footnotetext{
${ }^{23}$ Compte-rendu de la réunion de la CLE du SAGE Charente, 13-3-2012.

${ }^{24}$ Pierre Rosanvallon (2006, p. 15) évoque la formation d'une « défiance démocratique ».
} 
considérables d'un point de vue scientifique : un accès privilégié au terrain, notamment aux coulisses; mais comporte également des risques, dont celui d'être instrumentalisé pour légitimer une pratique. Néanmoins, il semble que son intérêt principal réside dans l'utilité sociale de la recherche, devenant ainsi un espace de réflexivité des acteurs de la participation vis-à-vis de leurs pratiques, pouvant contribuer à les ajuster. Héloïse Nez (2012) reprend ainsi la notion de public sociology, développée par Michael Burawoy (2005), pour l'appliquer aux recherches sur la démocratie participative. Ce dernier défend une sociologie non « confinée $»^{25}$ au monde académique, construite dans une relation dialogique avec un public et à destination de celui-ci. Mais encore faut-il trouver les instruments de diffusion de la recherche susceptibles de faciliter ce que Burawoy nomme «back-translation » (2005, p. 5), c'est-à-dire la restitution des savoirs produits à ceux dont ils proviennent. L'outil audiovisuel serait-il à même de jouer ce rôle?

\section{Traduire le vécu de l'expérience et les apprentissages qui en sont issus}

Pensé comme un condensé d'extraits d'entretiens réalisés avec quatre des douze participants $^{26}$, le film dure 25 minutes et a été monté sans commentaires ni voix off. Seuls quelques éléments d'information sont donnés par écrit au début et à la fin, et des images du bassin versant illustrent parfois les propos. Des titres structurent la progression du film, qui est à la fois chronologique (la mise en place du panel, le récit de l'expérience, puis les apprentissages et leçons tirées) et thématique. Il s'ouvre sur l'objet du débat, l'eau et l'agriculture intensive principalement. D'emblée le sujet apparaît sensible à travers les propos de Georges, l'agriculteur du groupe, qui dit s'être senti «attaqué » dans les débats. Ensuite sont évoqués les motivations et l'état d'esprit des participants avant le début du panel. Les différentes interventions montrent un intérêt certain pour la question de l'eau et une curiosité pour cette procédure originale, malgré le flou et les doutes quant à la capacité à répondre à la question posée. Les participants évoquent ensuite la construction progressive d'un groupe, le cadre et le rythme de travail, la délibération. Ces explications sont illustrées par des photographies prises lors des week-ends. Puis l'avis est brièvement présenté, et l'on comprend à travers les propos des participants que la conciliation des différents points de vue n'a pas été facile. Une partie du film évoque ensuite les apprentissages effectués lors de cette expérience, sur le fond du débat comme sur la forme. J'ai enfin choisi de consacrer une grande partie du film aux effets que la démarche a pu avoir sur les participants. La plupart disent être beaucoup plus attentifs qu'auparavant à la question de l'eau. Les interviewés font part de leurs réflexions sur le caractère démocratique ou citoyen de cette expérience. Dans leurs propos, l'implication et l'engagement sont particulièrement visibles.

L'introduction d'un processus participatif dans un domaine d'action publique aussi sectoriel et technique que celui de la gestion de l'eau questionne les possibilités de transformation de nos rapports au savoir et au pouvoir. Nous pouvons nous demander en quoi la «culture politique $\gg{ }^{27}$ et professionnelle spécifique à la gestion de l'eau peut se trouver questionnée, voire bouleversée, par la confrontation avec une toute autre culture politique, celle des citoyens dits « ordinaires » et «profanes » en la matière. Inversement, les rapports au savoir et

\footnotetext{
${ }^{25}$ Pour reprendre la distinction de M. Callon, P. Lascoumes et Y. Barthe (2001) entre « recherche confinée » et « recherche de plein air ».

${ }^{26}$ Sur les douze, seulement sept choisis aléatoirement ont été rencontrés en entretien en amont et à l'issue de l'expérience. Parmi eux, deux n'ont pas souhaité que l'entretien soit filmé, tandis que des imprévus techniques ne m'ont pas permis de filmer le troisième entretien.

${ }^{27}$ Expression utilisée par les anthropologues du politique pour désigner « un ensemble de manières de faire et de penser la politique partagées par une collectivité humaine » (Abélès, 1992, p. 23).
} 
au pouvoir entretenus par les citoyens connaissent-ils des changements au cours de la démarche et à son issue ? En confrontant deux types d'acteurs, la procédure favorise une meilleure interconnaissance et un processus d' "acculturation", car elle questionne les manières de faire et de penser des uns et des autres. Le film de recherche restitue l'expérience de l'intérieur et nous donne des éléments sur les effets qu'elle a pu avoir sur les participants. On distingue alors deux types d'apprentissages : d'une part, une «acculturation» à la thématique de l'eau et à sa gestion politique et, d'autre part, une "acculturation » à la délibération collective, exercice peu habituel pour la plupart des participants. Contrairement aux doutes dont ils font part au début quant à leurs capacités et compétences politiques, leurs conclusions témoignent finalement d'un processus de prise de confiance, assorti de l'idée selon laquelle, mis dans de bonnes conditions de réflexion, ils sont capables de produire une opinion de qualité sur un enjeu de société complexe. À la fin du film, chacun évoque ce qu'il attend de l'avenir de la gestion de l'eau sur le territoire, mais aussi des suites qui seront données à l'avis. Sur ce point, le film se clôt sur une note pessimiste et teintée d'un certain cynisme :

« Nathan: Je n'ai pas l'impression que ce genre de démocratie intéresse les gens [...] Non je ne suis pas optimiste, toujours pas, je ne l'étais pas au départ et je ne le suis pas à l'arrivée.

Marie : Après, est-ce qu'ils ont vraiment trouvé notre travail intéressant, complet ou je ne sais pas quoi... Est-ce que c'est pour nous faire plaisir ou est-ce que c'est vrai, je me pose la question. Qu'ils nous proposent de participer à la CLE, pareil, est-ce qu'on leur a dit "ça serait bien que vous le fassiez, ça fera plaisir aux gens, c'est la démocratie participative [elle mime des guillemets avec ses doigts], c'est le truc de Ségolène Royal [elle mime à nouveau], on est dans son fief, allons-y à fond"... Je n'en sais rien. »

Si on constate ainsi clairement des transformations du rapport au savoir, qu'en est-il pour le rapport au pouvoir, au politique? Le film montre que les participants sont sceptiques. Certes, plusieurs évoquent une expérience citoyenne et l'un d'entre eux va jusqu'à la comparer à ce qui se pratiquait dans l'Antiquité grecque en termes de démocratie. Mais cette remarque porte sur le cadre de la procédure en elle-même (notamment l'utilisation du tirage au sort) et n'évoque pas l'idée d'une réelle participation à la décision. Si l'un ou l'autre interprète le processus comme un passage progressif du statut de profane à celui d'expert, personne n'évoque l'idée d'une participation grandissante au pouvoir décisionnel. En ce sens, les effets possibles de cette expérience peuvent être contraires à ceux recherchés dans la mise en place de procédures participatives : «Loin de restaurer la confiance, les procédures de débats peuvent tout aussi bien aggraver la distance et le ressentiment lorsqu'elles sont mal menées » (Rui, 2004, p. 216).

\section{Le «feedback» du film, support de politisation du discours}

Avant d'envisager toute diffusion du film auprès des commanditaires de la démarche, il me fallait l'accord des personnes filmées. La première étape de ce protocole méthodologique fût donc la restitution auprès des panélistes seulement. Le feedback est une notion utilisée par l'ethnologue et cinéaste Jean Rouch $(1979$, p. 69) pour désigner ce moment de restitution du film ethnographique auprès des enquêtés, entraînant un dialogue fertile avec ces derniers autour de l'objet même de la recherche : 
"Cette extraordinaire technique du "feed-back" (que je traduirai par "contre-don audiovisuel") n'a certainement pas encore révélé toutes ses possibilités, mais déjà, grâce à elle, l'anthropologue n'est plus l'entomologiste observant l'autre comme un insecte (donc le niant) mais un stimulateur de connaissance mutuelle (donc de dignité). Le "feedback" relève donc à la fois de la méthode et de l'éthique du chercheur. »

Le feedback est certes un bon moyen de restituer la recherche, mais il constitue également un outil méthodologique précieux pour la prolonger. Au lieu de réduire l'enquêté à un objet d'étude, il le place dans une situation de dialogue avec le chercheur, en permettant d'approfondir les propos et d'adopter une position réflexive. J'ai donc mobilisé cette méthode afin de recueillir des réactions, impressions et remarques des participants au panel. Quelques mois après les entretiens filmés, je les ai invités à visualiser le film dans une salle de réunion mise à disposition par l'EPTB Charente à Saintes. Seulement six ont répondu présents, parmi eux les quatre personnes apparaissant dans le film. Après la visualisation, il y a eu un temps d'échanges, de relecture commune des passages du film. Ce moment a été l'occasion d'interprétations collectives de cette expérience, qu'aucun espace n'avait jusque-là permises. À travers l'échange avec ceux qui l'ont aussi vécu, le récit de l'expérience s'est transformé et enrichi de nouvelles dimensions. Par exemple, les propos de Nathan rapprochant cette expérience de l'idéal de démocratie grecque ont fait réagir Fabienne : "Après réflexion, c'est vrai que c'était le même principe. Quand on est juré au tribunal par exemple, c'est ça aussi. » Le feedback a ainsi ouvert une mise en débat de cette démarche, avec en toile de fond une réflexion sur les limites du système représentatif et une critique de la délégation de pouvoir à l'élu, «professionnel » de la politique, suspecté de servir aussi ses propres intérêts :

«Fabienne: Moi je dis que la CLE, elle devrait être constituée plus de panels d'habitants que de politiques, ça ferait peut-être bouger un peu plus. J'ai l'impression que ces gens-là, comme dit [Georges], c'est trop politique et pas assez...

Marie : Mais c'est quoi la différence entre un politique et un panel d'habitants pour toi?

Fabienne : Nous, on est plus proches de la réalité.

Marie : Oui mais les politiques c'est leur boulot. Nous sur douze on est quatre à être venus à la CLE, parce qu'on a autre chose dans notre vie, on a notre boulot. Et la personne qui finalement n'a pas de boulot à côté mais est payée pour le faire, c'est un politique. La seule différence c'est que le politique, il fait ça à plein temps.

Fabienne : Oui mais le politique il cumule les fonctions politiques, et moi je trouve qu'au bout d'un moment c'est l'étiquette qui compte et pas le reste. Donc ils ne sont pas aussi concernés que ça. $»^{28}$

Cet échange est révélateur de la conversion d'une expérience qui n'était au départ pas ou peu perçue comme politique, à une expérience politique à part entière. Mis à part Nathan, syndicaliste engagé que l'on pourrait caractériser comme l'individu le plus politisé du groupe, le caractère démocratique ou citoyen de cette expérience n'était généralement pas la première raison évoquée pour expliquer la volonté d'y participer. À l'issue de la démarche, on assiste à une requalification de cette expérience en termes plus politiques. Les échanges recueillis sont

\footnotetext{
${ }^{28}$ Cette citation et les suivantes sont issues du compte-rendu d'observation de la réunion de feedback avec les participants au panel du 29-08-2012.
} 
en effet révélateurs d'une "politisation» du discours, dans le sens d'une montée en conflictualité et en généralité (Duchesne, Haegel, 2001 ; Hamidi, 2006) : la conflictualisation suppose la reconnaissance de clivages sur un enjeu (ici le fonctionnement démocratique) et la montée en généralité se caractérise par le dépassement de l'anecdotique vers des principes généraux régissant la société. Alors que ces arènes sont parfois pensées comme des espaces de production du consensus, de neutralisation du conflit (Blondiaux, 2008, p. 138), les extraits précédents sont révélateurs d'une critique de la division du travail politique, potentiellement conflictuelle face aux tenants de la démocratie représentative. Sans aller nécessairement jusqu'à la formation d'un "contre-pouvoir » (Carrel, 2006), susciter la réflexivité sur une telle expérience peut donc conduire à une mise en question du fonctionnement démocratique. La montée en généralité s'est, quant à elle, manifestée par des propositions pour permettre une meilleure participation des citoyens aux "choses de la cité », comme celle d'instaurer quelques journées par an de «travail participatif» obligatoires pour tous (sur le modèle des jurys de tribunaux) :

« Marie : En Belgique, le vote est obligatoire, et pour ça il y a des journées de travail en moins, donc effectivement ça pourrait être instauré, le fait que deux ou trois jours par an, on n'ait pas de travail mais un travail participatif.

Fabienne: Ah oui, des journées citoyennes pour tous! Voilà, on a trouvé la solution!»

Au fil des échanges, les dimensions s'élargissent, passant de l'idée de panels d'habitants au sein de la CLE à des « journées citoyennes pour tous » concernant tous les domaines de la vie de la cité. Selon Luc Boltanski (1990, p. 74), « monter en généralité » nécessite de « s'extraire de la situation immédiate » pour se référer à l'intérêt général, au bien commun. Dans ces échanges, c'est l'organisation politique de la société dans son ensemble qui est visée. Lors de tels processus de participation, les citoyens ne deviennent pas seulement experts d'une thématique ou d'un enjeu, mais peuvent également apporter une "expertise d'usage » sur la question de la participation. Ayant vécu l'expérience de l'intérieur, connaissant les contraintes et obstacles qu'ils ont rencontrés (notamment la conciliation avec les autres sphères sociales), ils deviennent à même de produire des propositions qui pourraient y remédier. Certes, cette proposition relève sans doute d'une plaisanterie à laquelle ils ne croient pas réellement, ou du moins sur laquelle ils sont conscients de n'avoir aucune prise. Mais comme l'écrit Danilo Martuccelli (2004, p. 473), « les fantaisies, les rêveries, les rumeurs, les blagues, le renversement imaginaire des situations ("le monde-à-l'envers")» sont autant de manifestations d'une forme de «résistance » à la domination, qui peut prendre des formes beaucoup plus subtiles que l'expression d'une contestation directe.

La rencontre se clôt par la collecte des accords des quatre participants au film pour le diffuser auprès des commanditaires de la démarche. Ils me donnent tous leur accord sans difficulté. Comme s'ils étaient devenus eux-mêmes enquêteurs, une remarque m'est faite à propos de la restitution de ce film auprès de l'EPTB Charente : "C'est bien, ça pourrait les faire réagir, ils pourraient se dire "ah oui quand même, ils sont très pessimistes, il faudrait peut-être faire quelque chose !" » (Marie).

\section{Le film, un instrument de sensibilisation des acteurs politiques?}

Le film de recherche a-t-il eu les effets escomptés par Marie sur les commanditaires de la démarche ? Comme nous l'avons précédemment évoqué, aucun espace n'avait été prévu par 
les initiateurs de la démarche afin qu'ils se confrontent à l'évaluation par les citoyens du dispositif mis en place et de ses suites. La visualisation du film de recherche a donc constitué un substitut à cet espace. En octobre 2012, la directrice de l'EPTB Charente a organisé une séance de restitution de l'expérience auprès de l'ensemble de son équipe, dont seuls deux techniciens avaient travaillé à la mise en place. Les deux animateurs de l'Ifrée ont également participé à cette réunion, qui s'est déroulée en deux temps : la présentation de la démarche avec la visualisation du film institutionnel restituant l'avis, puis celle de mon film de recherche, à visée plus évaluative et réflexive. Comme l'a suggéré Marie, je pouvais raisonnablement $m$ 'attendre à une réaction face au scepticisme exprimé par les citoyens vis-àvis des suites de leur travail. Mais c'est tout un ensemble de prises de conscience qui a finalement mené à cette conclusion. En effet, il semble que le film ait permis une compréhension beaucoup plus fine de l'expérience, en particulier de la façon dont elle a été vécue par les participants. L'investissement et l'engagement exprimés de façon très sensible dans le film ont retenu l'attention des membres de l'EPTB et de l'Ifrée :

« Animateur Ifrée s'adressant à l'animatrice Ifrée : Tu avais senti ça toi, de l'extérieur ? On ne perçoit pas forcément à quel point ça peut les mobiliser. Je suis étonné de l'impact que ça a pu avoir sur eux, je les avais senti impliqués, intéressés, mais peutêtre pas dans la mesure de leur témoignage dans ce film.

Technicien EPTB : On a vraiment la sensation d'une expérience personnelle.

Animateur Ifrée : Oui, qu'il s'est passé quelque chose pour eux... $»^{29}$

En entrant «à l'intérieur », c'est-à-dire en allant au plus près du discours des participants, l'animateur a «senti » à son tour l'engagement vécu par ces derniers. À l'écoute de leurs témoignages, les commanditaires ont eu la «sensation » d'une expérience personnelle à part entière, formatrice, et pour laquelle les participants retirent une large satisfaction. Contrairement à un rapport écrit, le film dispose d'un atout, celui de permettre une compréhension par le sensible d'éléments difficilement saisissables par le recours à l'argumentaire. La prise en compte de l'affectif dans ce film, comme paramètre contribuant à mieux comprendre la façon dont cette expérience a été vécue, est source de prise de conscience chez les commanditaires. De plus, c'est la capacité du groupe à s'ériger en collectif, voire à parler au nom de l'intérêt général, qui a attiré leur attention. Face aux difficultés qu'eux-mêmes peuvent rencontrer dans la mobilisation des acteurs du bassin pour la construction du SAGE, l'engagement collectif exprimé ici revêt un caractère d'exemplarité :

«Ce que j'ai trouvé intéressant, [...] c'est l'apparition du "nous", qu'on trouve finalement dans plusieurs expressions. On n'est plus dans le "je", on est dans le "nous", dans la construction d'un collectif. Ça devrait nous interpeller sur la capacité de construire un "nous", et c'est un petit peu ce qu'il va falloir faire au niveau de la CLE » (Directrice EPTB Charente).

Frappés par l'implication et l'engagement, mais aussi le scepticisme exprimé à la fin du film, les échanges se sont ensuite portés sur ce qui a été fait de l'avis depuis la fin de la démarche :

\footnotetext{
${ }^{29}$ Cette citation et les suivantes sont issues du compte-rendu de la restitution du film de recherche auprès des commanditaires du 16-10-2012.
} 
«Directrice EPTB : Ça nous place devant nos responsabilités [...], la responsabilité qu'on a de quand même donner une suite.

Animateur Ifrée : Oui, et à la manière dont ils en parlent, ce n'est pas anodin pour eux. Ça parait important, ça serait une forme de reconnaissance du travail fait. »

Devant le constat que la CLE, qui travaille actuellement à élaborer l'état initial du SAGE, n'a pour l'instant pas rediscuté de l'avis citoyen, la directrice de l'EPTB a affirmé la nécessité de rester vigilant face au risque qu'il soit progressivement oublié. Ainsi EPTB et Ifrée décident à la fin de cette réunion de proposer au bureau de la CLE la formation d'un groupe de travail chargé de réfléchir spécifiquement à cette prise en compte, et plus largement à la façon dont on pourrait prolonger la participation des citoyens. Dans le but de mobiliser les membres de la CLE sur cette question, l'animateur de l'Ifrée propose même que le film leur soit montré, considérant qu'ils ne réagiront pas de la même manière devant la parole des citoyens, que si ces propos leurs sont rapportés : «Il y a des choses qui sont dites par les panélistes. Si c'est nous qui les disons, ça n'aura pas le même impact. » Le film est donc envisagé comme un outil de sensibilisation à même de faire réagir les membres de la CLE face à l'oubli provisoire de l'avis du panel de citoyens. En ce sens, il apparaît comme un instrument de traduction d'une implication et d'une attente à laquelle il serait irresponsable de ne pas répondre, au risque de renforcer le cynisme vis-à-vis du politique et des responsables politiques, et de produire ainsi l'effet inverse à celui initialement recherché.

\section{Conclusion}

L'analyse du contenu et de l'utilisation de ces deux films interroge les conditions dans lesquelles ce support peut contribuer à faire entendre la parole des citoyens, ou au contraire à perpétuer les asymétries de visibilité et de légitimité rencontrés avec les experts et acteurs politiques traditionnels. Le film produit par l'institution commanditaire de la démarche participative est apparu, de prime abord, comme un outil permettant de mieux faire entendre cette parole, au prix d'une réduction de la richesse de la délibération. Puis, l'analyse nous a amené à saisir l'influence de son utilisation dans le maintien des rôles politiques respectifs, permettant aux citoyens d'éviter l'inconfort d'une entrée dans le débat, et aux acteurs politiques de contenir la parole citoyenne et donc de ne pas avoir à la prolonger. Quant au film de recherche, il s'inscrit dans une volonté d'engager un processus réflexif sur la démarche participative dans son ensemble et non plus seulement sur la parole citoyenne qui en est le fruit. En cela, il contribue à mettre en lumière toute l'importance de l'expérience vécue par les participants. Et lors du feedback, cette expérience s'est enrichie d'une dimension politique encore peu exprimée. Enfin, vis-à-vis des acteurs politiques, ce film a suscité une compréhension plus sensible de l'expérience participative, portant particulièrement sur l'engagement des citoyens dans cette démarche. Il a ainsi contribué à relancer la réflexion sur l'avenir de l'avis citoyen qui avait jusque-là été quelque peu oublié. Indirectement, le film a été un support de sensibilisation et de responsabilisation des acteurs politiques vis-à-vis des suites qu'il reste à donner au travail des citoyens. Cela pourrait, à plus long terme, concourir à une réelle prise en compte de l'avis dans l'élaboration de la politique de gestion locale de l'eau en train de se construire, ce qui reste encore à vérifier. L'analyse a finalement montré que l'outil audiovisuel s'avère tout à fait intéressant pour répondre aux objectifs d'une « sociologie publique» dans les termes de Burawoy: en facilitant le processus de «backtranslation ", il provoque la réflexivité chez les acteurs. Les connaissances ainsi co-produites avec ces derniers peuvent, comme dans le cas présent, contribuer à modifier le cours des processus observés. 


\section{Bibliographie}

p. 15-30.

Abélès M., 1992, «Anthropologie politique de la modernité », L'Homme, 32 (121),

Anselme M., 2000, Du bruit à la parole. La scène politique des cités, La Tour d'Aigues, Éditions de l'Aube.

Barbier R., Bedu C., Buclet N., 2009, «Portée et limites du dispositif "jury citoyen". Réflexions à partir du cas de Saint-Brieuc », Politix, 2 (86), p. 189-207.

Bedu C., 2010, Quand une citadelle technique se (sou)met à l'"impératif délibératif ». Récit et analyse pragmatique d'une procédure de type "mini public » dans le domaine de l'eau potable, thèse pour le doctorat de sociologie, Université de Strasbourg.

Blondiaux, 2008, «Démocratie délibérative vs. démocratie agonistique ? Le statut du conflit dans les théories et les pratiques de participation contemporaines », Raisons politiques, 30 (2), p. 131-147.

Boltanski L., 1990, L'Amour et la Justice comme compétences, Paris, Métailié.

Bonaccorsi J., Nonjon M., 2012, «"La participation en kit" : l'horizon funèbre de l'idéal participatif », Quaderni, 79 (3), p. 29-44.

Bourg D., Boy D., 2005, Conférences de citoyens, mode d'emploi, Paris, Charles Léopold Mayer/Descartes \& Cie. 4-28.

Burawoy M., 2005, « For Public Sociology », American Sociological Review, 70 (1), p.

Callon M., 1984, «Éléments pour une sociologie de la traduction : la domestication des coquilles Saint-Jacques et des marins pêcheurs dans la Baie de Saint-Brieuc ", L'Année sociologique, 36, p. 169-207.

Callon M., Lascoumes P., Barthe Y., 2001, Agir dans un monde incertain. Essai sur la démocratie technique, Paris, Le Seuil.

Carrel M., 2006, « Politisation et publicisation : les effets fragiles de la délibération en milieu populaire », Politix, 75 (3), p. 33-51.

Duchesne S., Haegel F., 2001, «Entretiens dans la cité, ou comment la parole se politise », EspacesTemps. Les Cahiers, 76-77, p. 95-109.

Goodin R. E., Dryzek J., 2006, « Deliberative Impacts: The Macro-Political Uptake of Mini-Publics », Politics and Society, 34 (2), p. 219-244.

Gourgues G., 2012, "Avant-propos : penser la participation publique comme une politique de l'offre, une hypothèse heuristique », Quaderni, 79 (3), p. 5-12.

Hamidi C., 2006, «Éléments pour une approche interactionniste de la politisation. Engagement associatif et rapport au politique dans des associations locales issues de l'immigration », Revue Française de Science Politique, 56 (1), p. 5-25.

Lascoumes P., 2010, "Traduction », in L. Boussaguet, S. Jacquot, P. Ravinet (dir.), Dictionnaire des politiques publiques, Paris, Presses de Sciences Po, p. 632-640.

Martuccelli D., 2004, «Figures de la domination », Revue française de sociologie, 45 (3), p. 469-497.

Mazeaud A., 2012, «L'instrumentation participative de l'action publique : logiques et effets. Une approche comparée des dispositifs participatifs conduits par la région PoitouCharentes », Participations, 2 (1), p. 53-77.

Mermet L., 2007, «Épilogue. Débattre sans savoir pourquoi : la polychrésie du débat public appelle le pluralisme théorique de la part des chercheurs », in M. Revel, C. Blatrix, L. Blondiaux, J.-M. Fourniau, B. Hériard Dubreuil, R. Lefebvre (dir.), Le débat public. Une expérience française de démocratie participative, Paris, la Découverte, p. 368-380.

Nez H., 2011, «Nature et légitimités des savoirs citoyens dans l'urbanisme participatif. Une enquête ethnographique à Paris », Sociologie, 4 (2), p. 387-404. 
Nez H., 2012, « For a public sociology on participatory democracy. Reflexive feedback on research conducted in an association ", Revista International de Sociología, 70 (2), p. 185208.

Nez H., Talpin J., 2010, «Généalogies de la démocratie participative en banlieue rouge : un renouvellement du communisme municipal en trompe-l'œil ? », Genèses, 79 (2), p. 97-115.

Notte O., Salles D., 2011, «La prise à témoin du public dans la politique de l'eau. La consultation directive-cadre européenne sur l'eau en Adour-Garonne », Politique européenne, 33, p. 37-62.

Rancière J., 1998, Aux bords du politique, Paris, La Fabrique. Seuil.

Rosanvallon P., 2006, La contre-démocratie. La politique à l'âge de la défiance, Paris,

Rouch J., 1979, «La caméra et les hommes », in C. de France (dir.), Pour une anthropologie visuelle, Paris/La Haye/New-York, Mouton/EHESS.

Rui S., 2004, La démocratie en débat. Les citoyens face à l'action publique, Paris, Armand Colin.

Sintomer Y., 2007, Le pouvoir au peuple. Jurys citoyens, tirage au sort et démocratie participative, Paris, La Découverte.

Sintomer Y., 2008, « Du savoir d'usage au métier de citoyen ? », Raisons politiques, 31 (3), p. 115-133.

Talpin J., 2010, «Ces moments qui façonnent les hommes. Éléments pour une approche pragmatiste de la compétence civique », Revue française de science politique, 60 (1), p. 91115. L'Harmattan.

Topçu S., Cuny C., Serrano Velarde K. (dir.), 2008, Savoirs en débat, Paris,

\title{
Résumé
}

Cet article propose une analyse de l'utilisation de l'outil audiovisuel comme instrument de traduction original d'une expérience participative. Il explore l'utilisation de deux films réalisés à l'issue d'un panel de citoyens sur la gestion de l'eau : un film « institutionnel », et un film de recherche. L'attention est portée sur ce que la «mise en film » fait à la parole citoyenne, mais aussi au film comme support de réflexivité des acteurs vis-à-vis de leurs pratiques. L'analyse s'inscrit dans une réflexion globale sur le rôle de cet outil dans la (re)définition des rapports de pouvoir à l'œuvre dans les processus de démocratie participative.

\section{Mots clés}

Démocratie participative, film, traduction, restitution, effet, politiques de l'eau.

\section{Title}

Making citizens' voice heard. An analysis based on two films about a citizen's panel on water management.

\begin{abstract}
This article investigates how an audiovisual tool can be used as an original feedback instrument for a participatory experience. It explores the use of two films during a citizens' panel on water management: an "institutional" film and a research film. Attention is drawn to
\end{abstract}


what the process of "putting into film" does to the citizens' voice. The article also explores the use of film as a support for actors' reflexivity on their own practices. This analysis takes place in a broader reflection on the role of films in the (re)definition of the power relationships at work in participatory processes.

\section{Keywords}

Participatory democracy, film, translation, feedback, effect, water policy. 